\title{
Predicting Students' Course Performance Based on Learners' Characteristics via Fuzzy Modelling Approach
}

\author{
Chee Siong The ${ }^{\#}$, Shu Hsien Lee ${ }^{\#}$ Mohamad Hardyman Barawi ${ }^{\#}$ \\ ${ }^{\#}$ Faculty of Cognitive Sciences and Human Development, Universiti Malaysia Sarawak, 94300 Kota Samarahan, Sarawak, Malaysia \\ E-mail: csteh@unimas.my; shlee_pg@hotmail.com;bmhardyman@unimas.my
}

\begin{abstract}
Frequent assessment allows instructors to ensure students have met the course learning objectives. Due to lack of instructor-student interaction, most of the assessment feedbacks and early interventions are not carried out in the large class size. This study is to proposes a new way of assessing student course performance using a fuzzy modeling approach. The typical steps in designing a fuzzy expert system include specifying the problem, determining linguistic variables, defining fuzzy sets as well as obtaining and constructing fuzzy rules is deployed. An educational expert is interviewed to define the relationship between the factors and student course performance. These steps help to determine the range of fuzzy sets and fuzzy rules in fuzzy reasoning. After the fuzzy assessing system has been built, it is used to compute the course performances of the students. The subject expert is asked to validate and verify system performance. Findings show that the developed system provides a faster and more effective way for instructors to assess the course performances of students in large class sizes. However, in this study, the system is developed based on 150 historical student data and only a total of six factors related to course performance are considered. It is expected that considering more historical student data and adding more factors as the variables help to increase the accuracy of the system.
\end{abstract}

Keywords — assessment feedback; fuzzy logic; fuzzy inference system; student modeling; intelligent tutoring system.

\section{INTRODUCTION}

Examining learner characteristics is one of the crucial principles in instructional design [1]. Insights on learner characteristics when designing instruction and learning help to assure that most of the target audience is receptive to the instruction. It also often leads to effective, efficient, and appealing teaching and learning.

An instructional designer should determine the learner's skills, knowledge, and attitude toward learning before designing the instruction. Among the characteristics that should be taken into consideration include specific prior knowledge, prerequisite knowledge, academic motivation, attitudes and levels of education and/or ability [1]. Learner analyses are often done via self-reported instruments [2], [3]; surveys [4], [5]; as well as interview [6].

Some studies use statistical methods to predict student performance and to examine factors that are associated with student performance [7], [8]. Other methodologies are later employed to perform such prediction. Recent studies such as [9] use eye-gaze activities to examine how individuals perform different gazing activities when holding different team roles in pair programming and derive predictive insight into their post-test performance. Data mining techniques that employ classification algorithms like Decision Tree, Naïve
Bayes and Support Vector Machine were used to discover the significant predictors of student's performance [10]. Such prediction enables teachers and parents to keep track of student performance and provide appropriate intervention. Some studies apply genetic programming to predict online student performance and investigates the efficacy of Singular Value Decomposition (SVD), a matrix factorization technique, for predicting student performance [11], [12].

To provide a better analysis of students' learning characteristics, student modelling is to be built. Student modelling is defined as the process of creating representation of a student's characteristics [13]. As the characteristics involve many factors and facts that are vague and unmeasurable, student modelling should be able to deal with such uncertainty [14]. One possible method to handle such uncertainty is by using fuzzy logic. Fuzzy logic was introduced by Lofti Zadeh in 1965 as an approach for computing with words in which words are used in place of numbers for computing and reasoning. This approach tackles ambiguous problems caused by human subjectivity. By using fuzzy modelling approach, an expert's knowledge in verbal descriptions can be transformed into mathematical models through computer algorithms [15].

Studies also reveal the various factors that influence a student's course performance, such as socio-economic, psychological, environmental and individual factors [16]. 